\title{
DIAGNOSTICS AND CORRECTION OF THE ELECTRON BEAM TRAJECTORY IN THE CORNELL WIGGLER AT THE ACCELERATOR TEST FACILITY
}

\author{
V. Sajaev, ANL, Argonne, IL, Li-Hua Yu, A. Douryan, R. Malone, X. Wang, V. Yakimenko, \\ BNL, Upton, NY
}

\begin{abstract}
High-Gain Harmonic Generation experiment is underway at the ATF. This experiment utilizes $41 \mathrm{MeV}$ electron beam produced by photocathode electron gun and s-band linac. The strict requirements on electron beam trajectory in a $2-\mathrm{m}$ long, 60 period undulator are essential for the success of the experiment. The simulation demands that trajectory errors should be less than $100 \mu \mathrm{m}$ in the wiggler. Five pop-in monitors are used to measure the electron beam trajectory along the wiggler. HeNe laser defines reference line. We present our results and experience concerning different aspects of the electron beam alignment.
\end{abstract}

\section{INTRODUCTION}

Currently, a high gain harmonic generation experiment is being commissioned at the Accelerator Test Facility at BNL. This experiment is based on collaboration between BNL and the APS. A brief description and status of the experiment can be found in these proceedings [1].

One of the important requirements of the experiment is the electron beam trajectory tolerance inside the radiator - Cornell wiggler. Using multi-particle simulations with the TDA-3D code the gain as a function of the transverse rms displacement of the trajectory was studied [2]. Based on these simulations it was concluded that the displacement of the trajectory in the wiggler should be less than $100 \mu \mathrm{m}$. In this paper we present the details of the beam trajectory measurement and correction in the Cornell wiggler.

\section{BEAM LINE DESCRIPTION}

The experiment layout consists of two wiggler magnets separated by dispersive section. First wiggler in combining with $\mathrm{CO}_{2}$ laser radiation provides energy modulation of the electron beam. Dispersive section transforms the energy modulation to spatial bunching. Upon passing through the third wiggler (the Cornell wiggler) the prebunched electron beam radiates coherently. Main parameters of the Cornell wiggler are presented in the table below.

\begin{tabular}{|l|c|}
\hline Undulator Period & $3.3 \mathrm{~cm}$ \\
\hline Number of Periods & 60 \\
\hline Peak Magnetic Field & $0.467 \mathrm{~T}$ \\
\hline
\end{tabular}

The horizontal trajectory of the beam calculated using measured magnetic field is shown on Figure 1. The averaged electron trajectory in the Cornell wiggler has offset of about $800 \mu \mathrm{m}$ relative to the axis of the beam line.

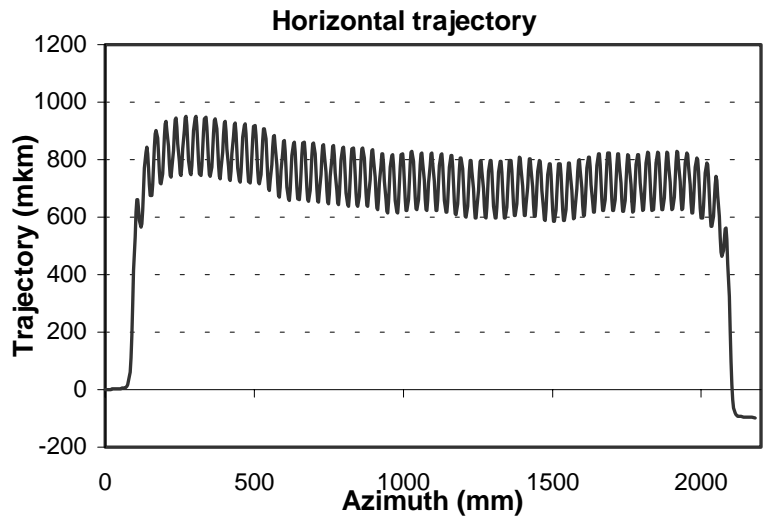

Figure 1 Horizontal trajectory in the Cornell wiggler calculated using measured magnetic field.

The gain of FEL depends on maintaining a precise phase relationship between the wiggle motion and the electric field of the radiation. It is necessary to effectively control the transverse betatron oscillations to satisfy this relationship. In a wiggler of conventional design there is no focusing in horizontal plane so external focusing is required. This focusing is provided by four-wire correctors mounted on the vacuum chamber inside the magnetic gap of the Cornell wiggler. Four separate corrector sections are distributed over the wiggler. These correctors can provide both dipole and quadrupole fields. The strength of the quadrupole correctors is adjusted to keep horizontal and vertical beta functions approximately constant along the wiggler and equal to $0.6 \mathrm{~m}$. Lattice functions of the beam line are shown on Figure 2.

\section{ELECTRON BEAM DIAGNOSTICS}

Electron beam diagnostics are provided by pop-in monitors. There are 8 pop-in monitors along the beam line. Five of them are in the Cornell wiggler. These use coaxial actuators because access to the vacuum chamber is limited to only one side. Fluorescent screens are used for imaging. Each pop-in monitor is equipped with own CCD camera and can be connected to a frame grabber. 
The CCD cameras and optics provide resolution of $10 \mu \mathrm{m}$.

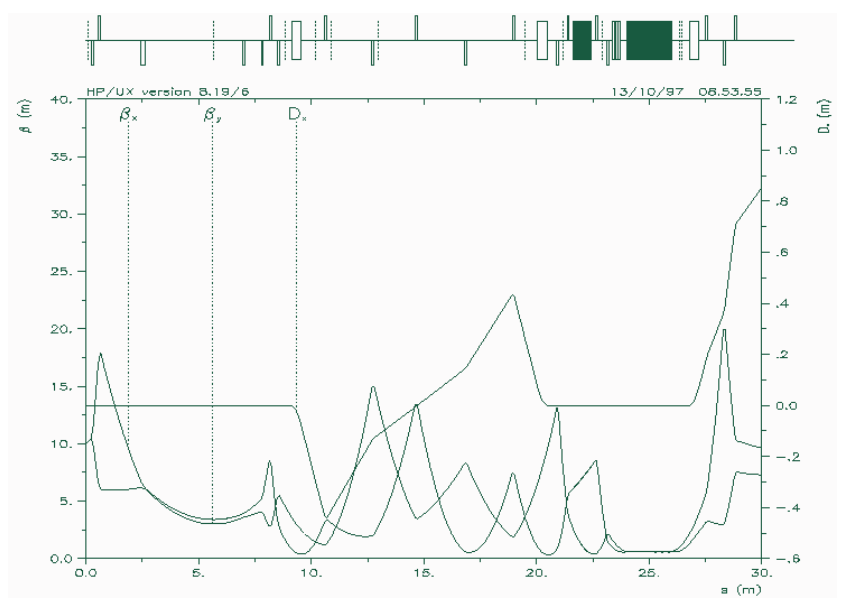

Figure 2 Beta functions of the beam line. First and third wigglers are black boxes.

A HeNe laser, which is aligned using two irises, defines the reference straight line. The HeNe laser beam position strongly depends on the temperature of the laser. For our case 1 degree $\mathrm{C}$ approximately corresponds to $20 \mu \mathrm{m}$ displacement at the pop-in monitor locations. There is no temperature stabilization for the $\mathrm{HeNe}$ laser so this dependence should be always kept in mind.

The HeNe laser is also used for calibration of the pop-in monitors. The laser beam is deviated with rotating thick glass, and frame grabber readings are compared with known laser displacement. It was found that the calibration is not constant along the monitor area, so we suppose that aluminium mirrors inside the vacuum chamber are not flat.

The HeNe laser beam is focused to have a wedge on $3^{\mathrm{d}}$ monitor of the Cornell wiggler. The distance between focusing telescope and the $3^{\mathrm{d}}$ monitor is about $10 \mathrm{~m}$ so the minimum possible beam size at that point is about 1 $\mathrm{mm}$. On other pop-in monitors the laser spot a bit larger. Such big laser beam size places high demands on laser beam and pop-in monitor optics quality because it is necessary to determine the position of the centroid of the $1 \mathrm{~mm}$ spot with accuracy of order of ten microns.

\section{TRAJECTORY CORRECTION}

Magnetic measurements of the Cornell wiggler has demonstrated that the real magnetic field of the wiggler does not have considerable distortions and corresponds well to the designed values. So one can expect that the trajectory of the electron beam with initial coordinates corresponding to the focusing axis of the wiggler should be straight line. Therefore the problem of the trajectory correction in the Cornell wiggler is to find the initial conditions for the electron beam corresponding to the focusing axis. The HeNe beam, which design position should coincide with the focusing axis, defines this axis.
Despite the precise positioning of the irises used for the $\mathrm{HeNe}$ laser alignment, it turned out that the electron beam trajectory with the same initial conditions as the laser beam is not straight but has betatron oscillations. This means the HeNe laser beam position is not coincident with the focusing axis of the Cornell wiggler. In such situation, to correct the trajectory it is necessary first to determine the position of the HeNe laser beam relative to the focusing axis. To find this we assume that the HeNe laser beam is a straight line but with unknown position at the entrance of the wiggler. Then we measure difference between the electron beam trajectory and the $\mathrm{HeNe}$ laser beam for each pop-in monitor in the wiggler. After that we use calculated beam transfer matrices to fit the betatron oscillations of the electron beam. The variables for this fit are position and angle of the $\mathrm{HeNe}$ beam and angle of the electron beam at the entrance of the wiggler. The initial position of the electron beam can be found as sum of the $\mathrm{HeNe}$ beam position and measured distance between laser and electron beam at the first pop-in monitor. A least square fit procedure is used to determine the HeNe offset and the electron beam entrance coordinates. The examples of the measured and fitted vertical trajectories are presented on Figure 3. The vertical offset between the HeNe laser beam and the focusing axis is about $400 \mu \mathrm{m}$.

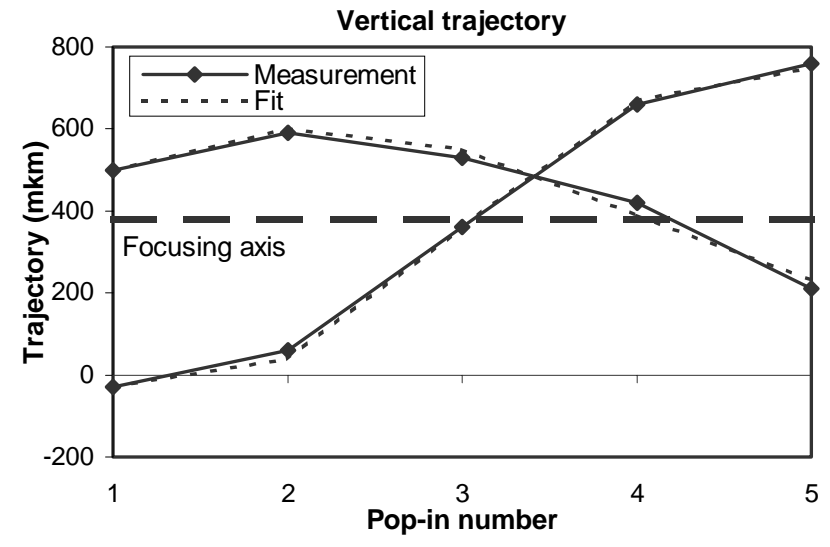

Figure 3 Examples of measured and fitted vertical trajectories. Focusing axis is also shown. The offset between the HeNe laser and the focusing axis is about $400 \mu \mathrm{m}$.

After the HeNe offset is determined, the electron beam can be moved to the focusing axis using only correctors outside the wiggler. This procedure is implemented on Labview, and results of correction of the vertical trajectory are shown on Figure 4. Maximum errors for the corrected trajectory are $\pm 50 \mu \mathrm{m}$, which satisfies the requirements.

Same technique can be applied to horizontal trajectory but there are few obstacles that make it more difficult. According to Figure 1 the trajectory on first and fifth pop-in monitors (which are situated right before and after the wiggler) lies on a beam line axis, while on other 
three monitors it lies in the wiggle motion region with $800 \mu \mathrm{m}$ average offset. Longitudinal positions of these three monitors with respect to the wiggle motion are also known with moderate accuracy. That's why it is useful to have trajectory measurement not relying on the $\mathrm{HeNe}$ laser.

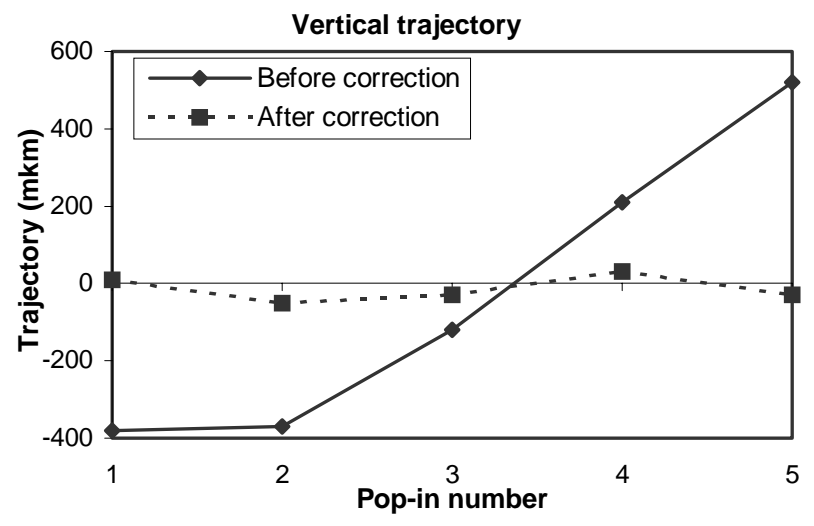

Figure 4 Vertical trajectory before and after correction.

The main purpose of the HeNe laser in our case is to define zeros of the pop-in monitors. Without the laser another definition of zeros is necessary. In the magnetic field of the wiggler there is no natural focusing in the horizontal plane so the only candidate for the zeros definition is the focusing axis of the 4-wire correctors.

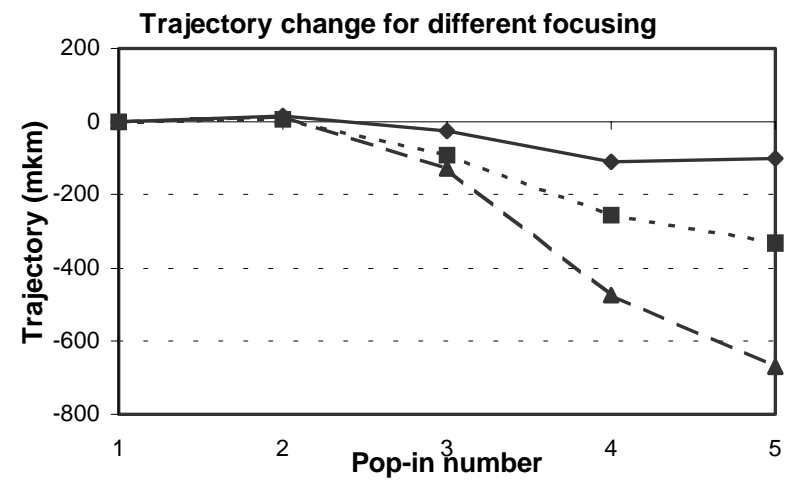

Figure 5 Relative trajectory change due to focusing variation.

To find the trajectory without the HeNe laser we vary the focusing in the Cornell wiggler and measure the corresponding beam position changes. Example of these measurements for different focusing is presented on Figure 5. The difference in beam trajectories for various focusing in the wiggler depends on the focusing properties of the wiggler and initial coordinates of the electron beam. Comparing measured beam behaviour with calculated one the unknown beam position at the entrance of the wiggler can be found.

Least squares are used to fit measured data and to find position and angle of the electron beam at the entrance of the wiggler. A singular value decomposition method is used to find inverse matrix in the least squares. The results of the trajectory correction are shown on Figure 6 . The maximum errors of the corrected trajectory in this method are $\pm 30 \mu \mathrm{m}$ in both horizontal and vertical planes.

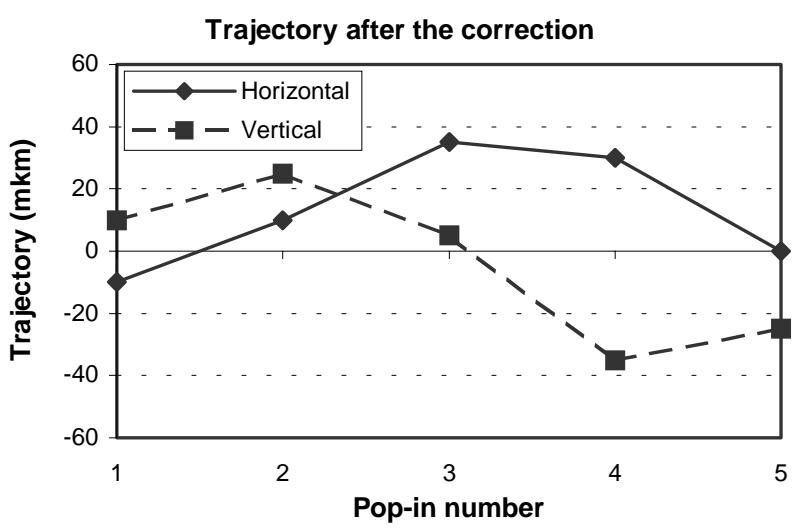

Figure 6 Results of the correction using the focusing variation for horizontal and vertical planes.

\section{CONCLUSION}

We present the electron beam diagnostics and the trajectory correction schemes for the HGHG experiments. The experiment requires the beam trajectory in the wiggler magnets to be straight line within $100 \mu \mathrm{m}$, and our correction schemes allow achieving such accuracy. However using HeNe laser as a reference line demands surprisingly high quality optical elements in pop-in monitors to achieve same accuracy as in methods using focusing variation in the wiggler.

In our case the focusing variation method demonstrates better accuracy of correction than the first method relying on the HeNe laser. The main problem of the first method seems to be in the determination of the $\mathrm{HeNe}$ beam positions. Due to long distance between telescope, which focus the $\mathrm{HeNe}$ beam, and pop-in monitors the minimum possible laser beam size on the monitors is about $1 \mathrm{~mm}$. To get good correction it is necessary to determine the HeNe beam position with accuracy of order of $10 \mu \mathrm{m}$. But non-ideal optical elements (telescope, mirrors, filters, etc.) cause small laser image distortions which result in the errors in measuring of the positions of the HeNe beam centroid. Therefore the laser beam trajectory is not a straight line any more, and this defines limitation for correction scheme.

\section{REFERENCES}

[1] L.-H. Yu at al, "The status of the high gain harmonic generation free electron laser experiment...", these proceedings.

[2] L.-H. Yu, "Design parameters of the high gain harmonic generation experiment using Cornell undulator A at the ATF", BNL-65983, informal report. 